\title{
IN SITU COMMUNITY CONTROL OF THE STABILITY OF BIOREDUCED URANIUM
}

\author{
David C. White ${ }^{1}$, Phillip E. Long², James P. McKinley² \\ Aaron D. Peacock ${ }^{1}$ and Yun-Juan Chang ${ }^{1}$
}

${ }^{1}$ Center for Biomarker Analysis, University of Tennessee, Knoxville, TN, USA ${ }^{2}$ Pacific Northwest National Laboratory, Richland, WA, USA 


\section{We could not do this work without our excellent collaborators!}

Kelly P. Nevin, Regina T. O’Neil, Helen Vrionis, Irene Ortiz Bernad, and Derek R. Lovley

\section{UMASS}

Richard Dayvault

S.M. Stoller Corp.

\section{Darrell Chandler}

ANL 


\section{Shift of viable biomass \& community composition by acetate addition to groundwater UMTRA site}
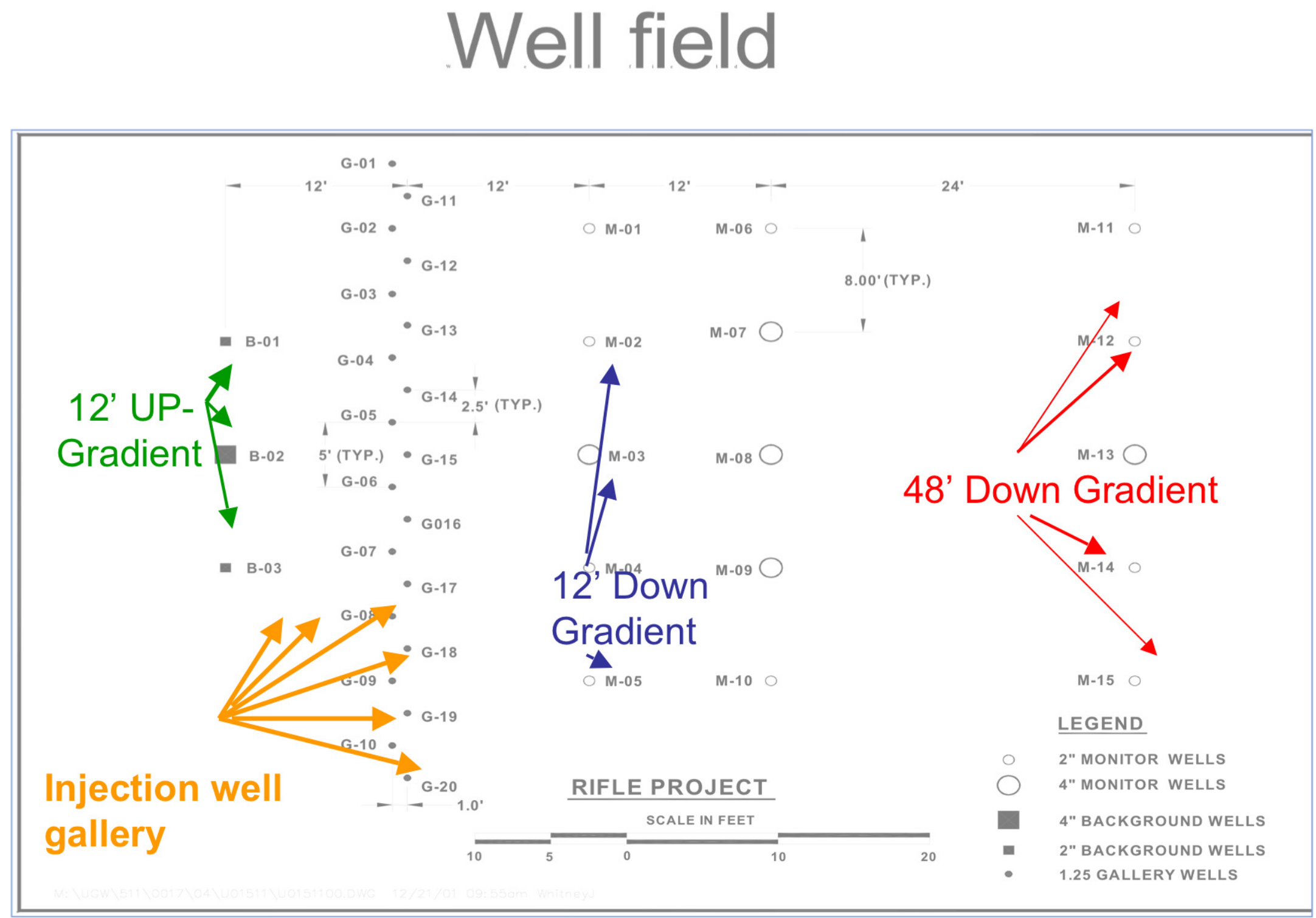


\section{UMTRA Old Rifle site}

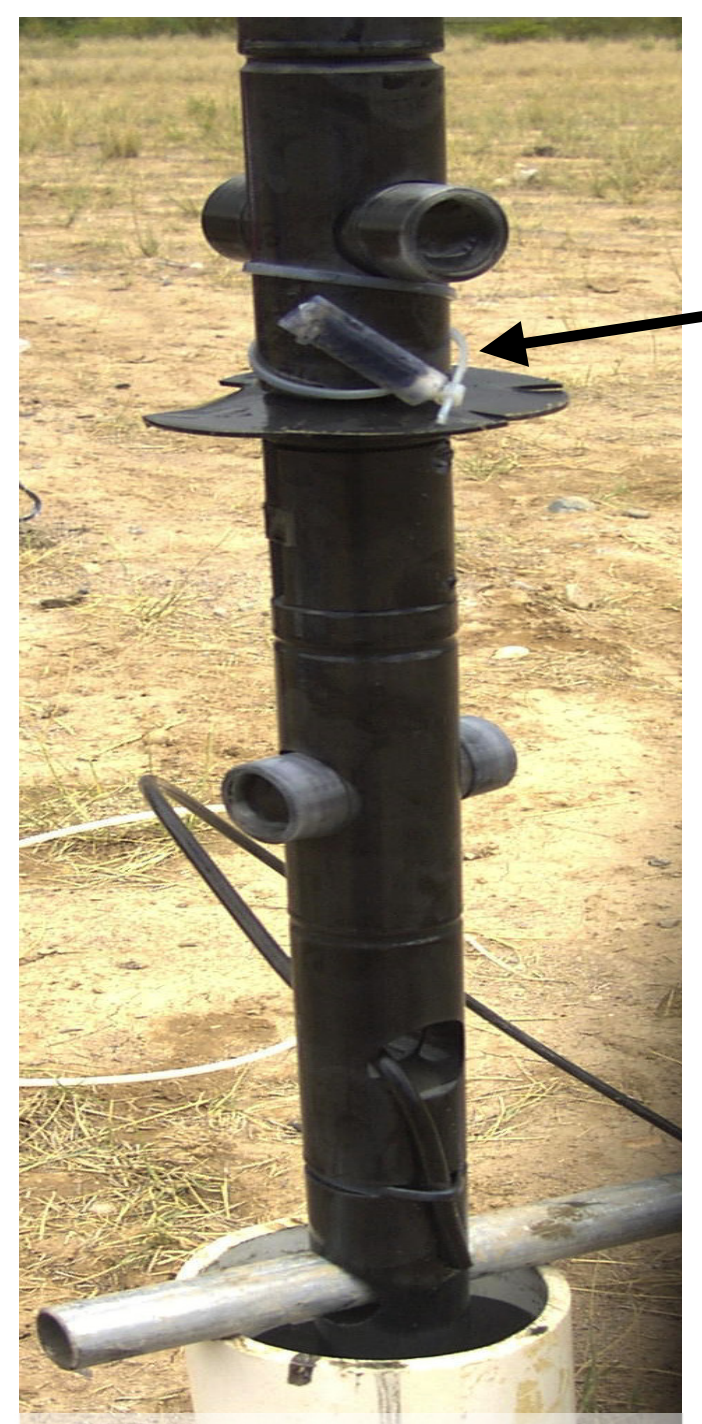

Multi-level Sampler with Bead coupon
Sampler with

Beads

CLSM image of microbial colonization live/dead stain

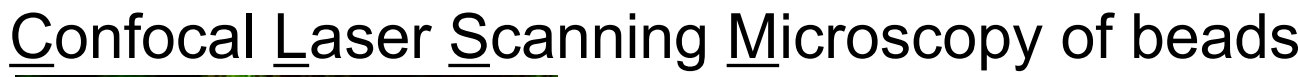

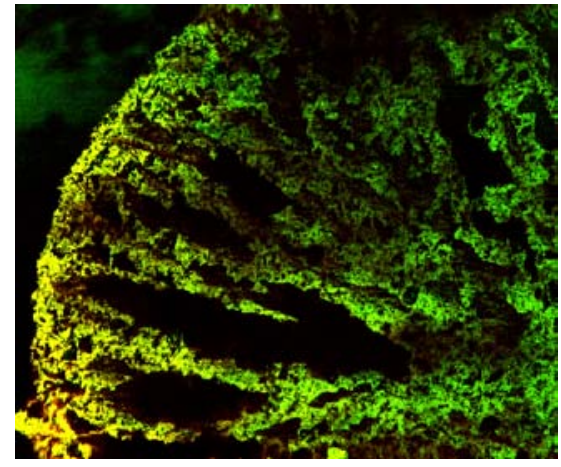
CLSM cross section of bead showing surface area for colonization

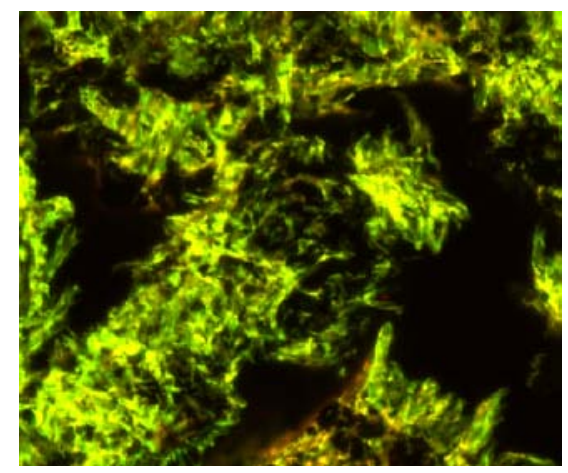
CLSM close-up of $\checkmark$ cross section

SEM of microbial colonization of glass wool

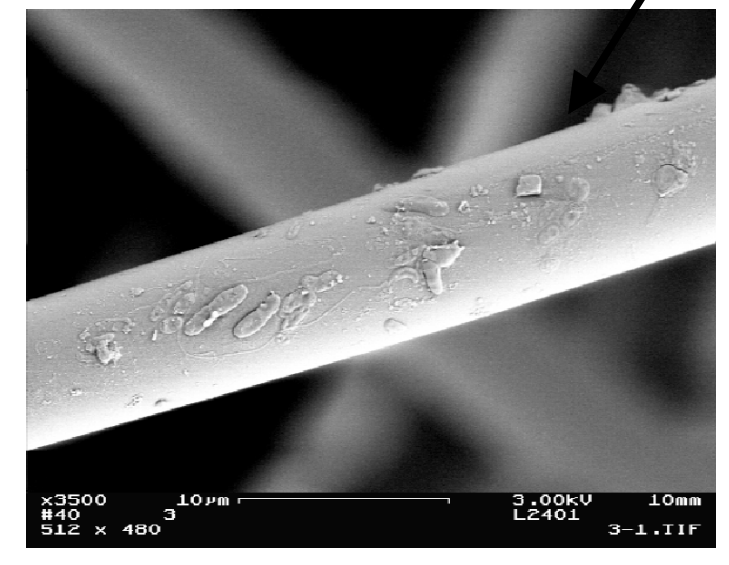




\section{Acetate Infusion induced}

A. Changes in the mineralogy

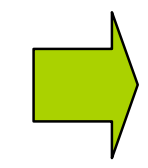

$\mathrm{FeS}_{0.9}$

B. Changes in the microbial community

Program is to test hypotheses as to How changes in A \& B Maintained the decrease in $\mathrm{U}(\mathrm{VI})$ in the groundwater

\section{TEST}

Monitor $\mathrm{U}(\mathrm{VI})$ loss in-well sediment incubators different mineral \& microbiological amendments 


\section{Assessing Subsurface Microbiota}

\section{Collecting Microbes}

\section{Bead Coupons}

Must colonize from water Grab Sample

Actively Growing

${ }^{13} \mathrm{C}$ in DNA \& PLFA

Biomass Intermediate

Lowest
Sediment

Disruptive

Includes slow \& non

Growing

Complexity Increases

Highest

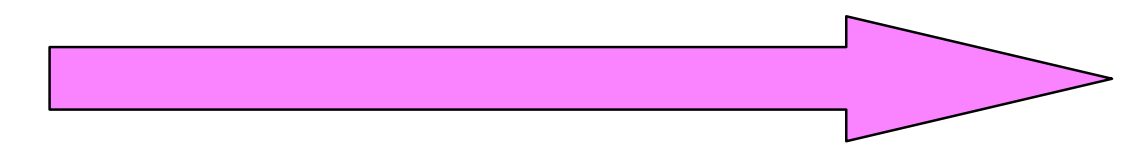


Why did $\mathrm{U}(\mathrm{VI})$ decrease in groundwater persist 7-9 months after acetate infection stopped? (Bead Coupons)

Up-Gradient 12' Down-Gradient 24' Down-Gradient 48' DownGradient

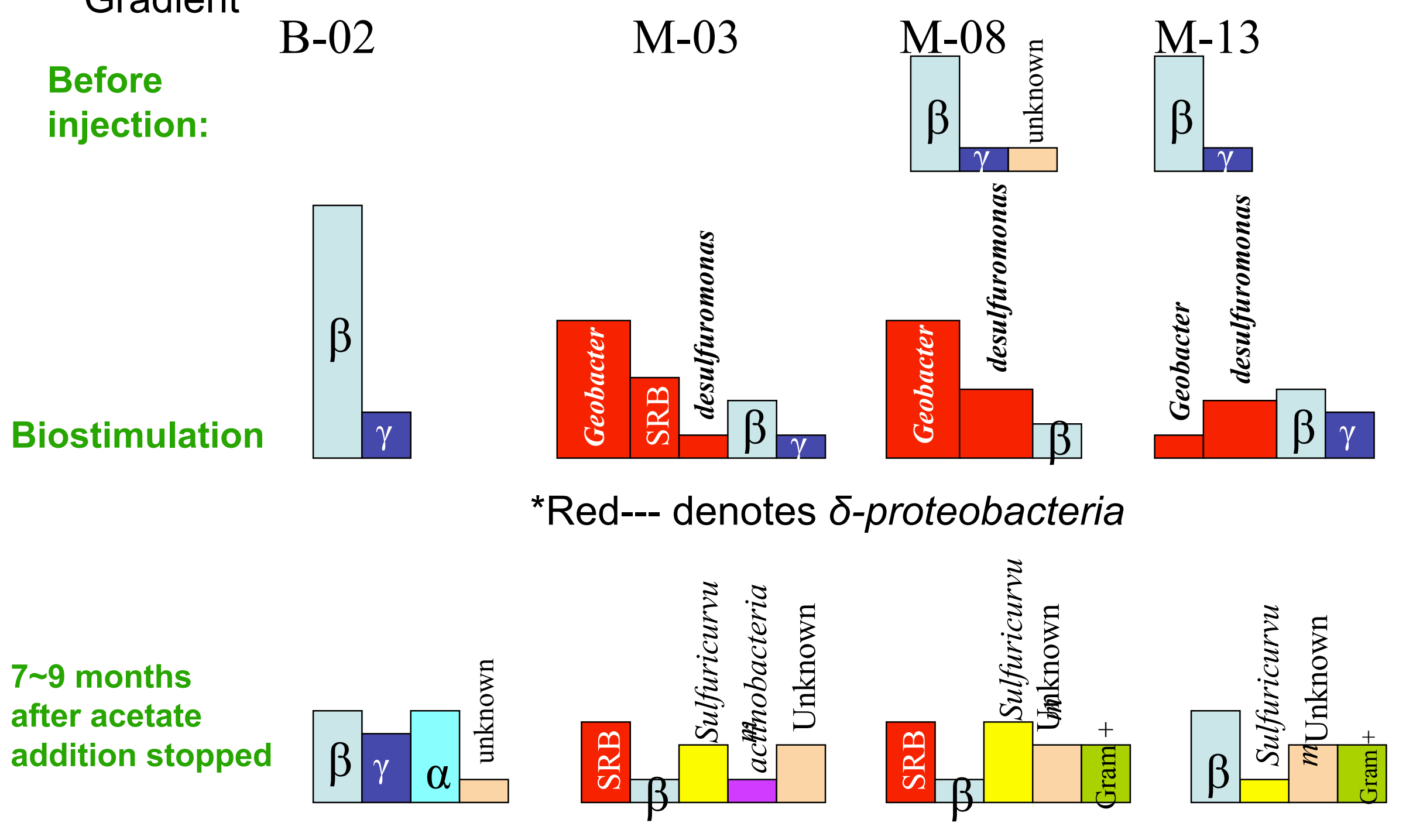




\section{Preliminary data (DGGE from Bead Coupons) suggests:}

Up gradient \& before injection stable community dominated by $\beta$-protrobacteria (Hydrogenophaga, Dechloromonas, Rhodoferax, Ferribacterium, Rhodocyclus, Methylophilus, Azoarcus, Y-Proteobacteria Pseudomonas

A Gram-negative, heterotrophic, carbon-limited, (high

cyclo/monoenoic PLFA), facultative-anaerobic, oxygenated (UQ/MK 2), utilizing refractory organics

Infusion Geobacter, Desulfuromonas/Pelobacter DIRB, $\delta$ Proteobacteria Desulfobacter, $\beta$-protrobacteria Ferribacterum, $\gamma^{-}$ Proteobacteria Pseudomonos,

\section{Rapid Biostumulation DIRB,SRB goes anaerobic \& growth stimulated}

7-9 months later , Sulfuricurvum, $\beta$-\& $\gamma$-Proteobacteria, Gram-positive Clostridia, $\delta$-Proteobacteria SRB

Sulfur oxidizing bacteria use sulfide to $\Rightarrow$ reduce Nitrate/ Oxygen maintain anaerobic status UQ/MK 06-0.2 \& SRB form U(IV)? 


\section{Hypotheses:}

1) After stimulation $\mathrm{Fe}(\mathrm{III})$ terminal electron acceptor non sulfate-reducing DIRB will be leave or be out-competed by more versatile microbes ? SRB

2) SRB \& Sulfur oxidizing bacteria play a critical role in the posttreatment maintenance of bio-reduced uranium by directly reducing $\mathrm{U}(\mathrm{VI})$, generating $\mathrm{H}_{2} \mathrm{~S}, \mathrm{HS}^{-}$and/or $\mathrm{FeS}_{0.9} \Rightarrow$ oxygen sinks maintaining $\mathrm{U}(\mathrm{IV})$.

3) Bioprecipitated amorphous $\mathrm{FeS}_{0.9}$ in sediments will maintain low $U(\mathrm{IV})$ reoxidation rates under conditions of low biomass, especially in presence of sulfur oxidizing bacteria

but $\mathrm{FeS}_{0.9}$ by itself is not sufficient to remove $\mathrm{U}(\mathrm{VI})$ from groundwater by abiotic reduction 


\section{Conceptual Model}

Start Acetate to C-starved $\beta$-proteobacter, oxygenated $\Rightarrow$ Anaerobic $\Rightarrow$ Geobacter + DIRB $\Rightarrow$ reduce $\mathrm{Fe}(\mathrm{III}) \Rightarrow$ great increase biomass $\Rightarrow$ Lo $\Rightarrow$ cal $\mathrm{Fe}(\mathrm{III})$ surfaces all reduced $\Rightarrow$ wave Geobacter (+ DIRB) moves distally

Continued acetate $\Rightarrow$ SRB increase in diversity (DSR) \& biomass $\Rightarrow$ produce $\mathrm{HS}^{-} \Rightarrow \mathrm{HS}^{-}+\mathrm{Fe}(\mathrm{II}) \Rightarrow \mathrm{FeS}_{0.9}$

Stop Acetate Biomass Cell lysis feeds Heterotrophs Gram-positive Clostridia + Desulfotomaculum (+ SRB) $\Rightarrow$ reduce $\mathrm{U}(\mathrm{VI})$ \& produce acid carbonate \& $\mathrm{U}(\mathrm{VI})$ complex

Sulfur Oxidizing (Sulfuricurvum) use $\mathrm{HS}^{-}$to reduce $\mathrm{NO}_{3} \& \mathrm{O}_{2}$ Without $\mathrm{NO}_{3} \& \mathrm{O}_{2}$ Dechloromonas \& Geobacter not reoxidize $\mathrm{U}(\mathrm{IV})$

Does Cigar Lake U mine deposit have high $\mathrm{FeS}_{0.9}+\mathrm{SOB}$ \& low UQ/MK? 


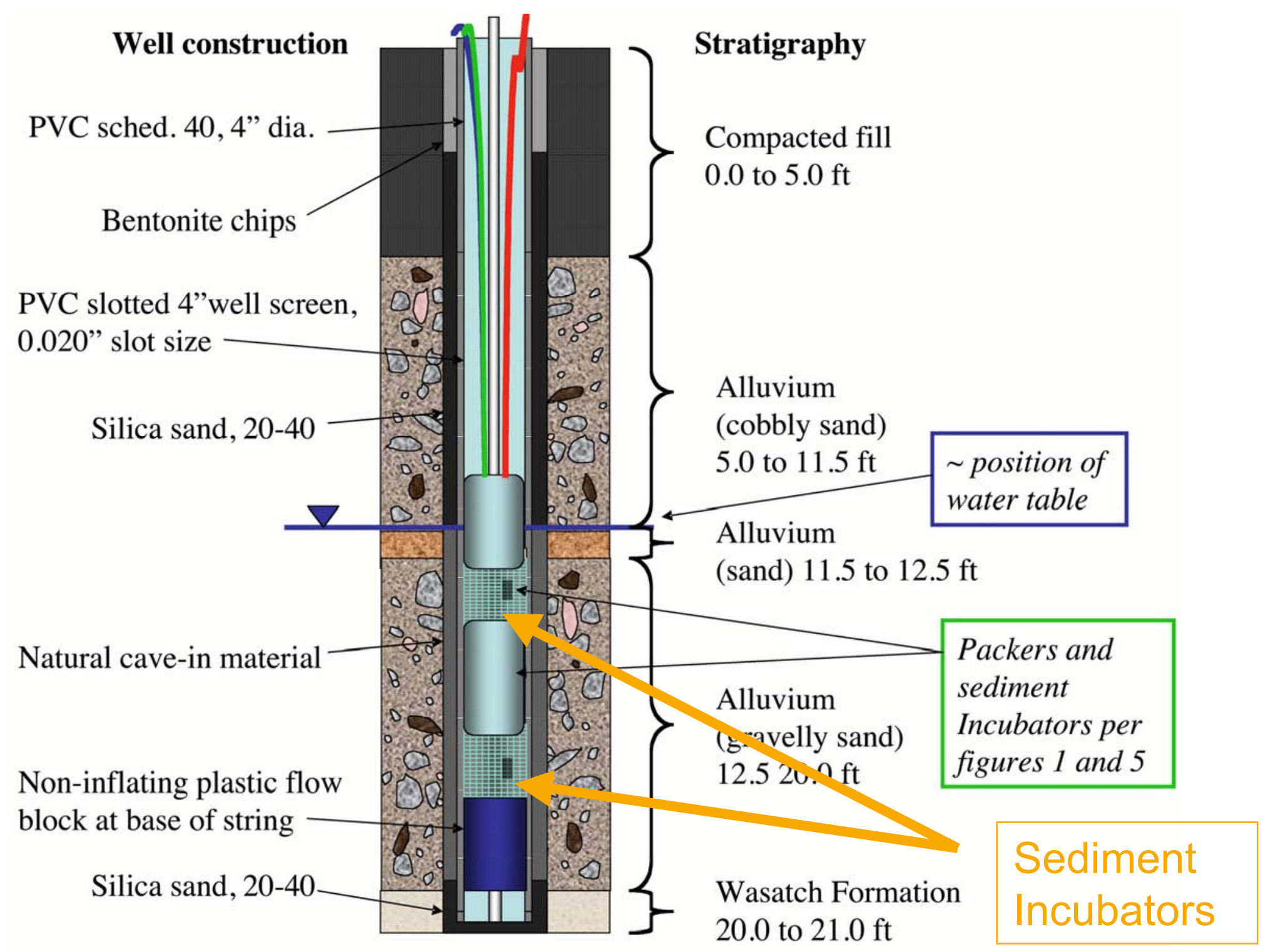

Figure 4. Proposed emplacement of two sediment incubators in a typical 4" well (B-02, Old Rifle UMTRA Site). 


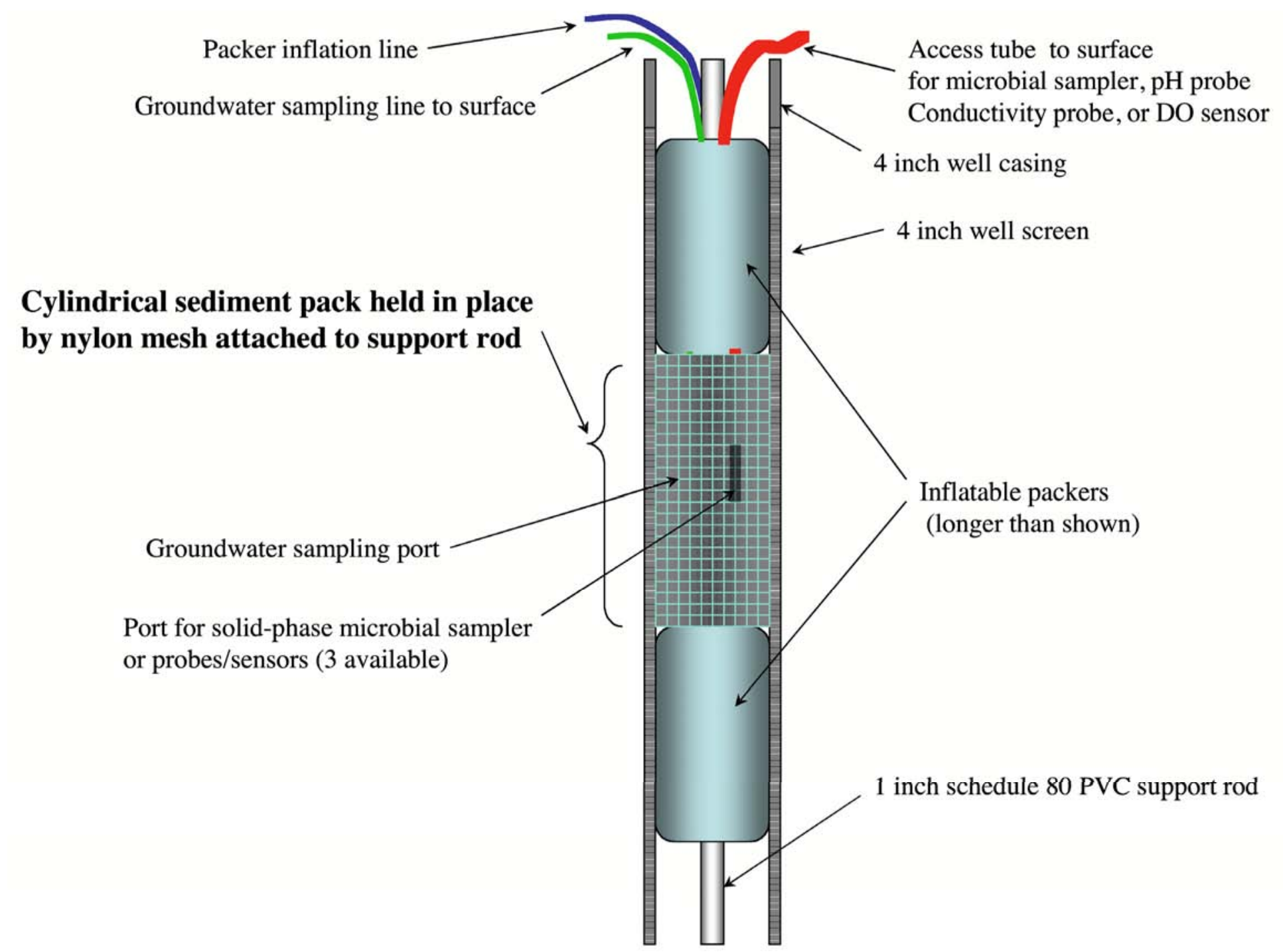

Figure 1. Conceptual design of sediment incubator 


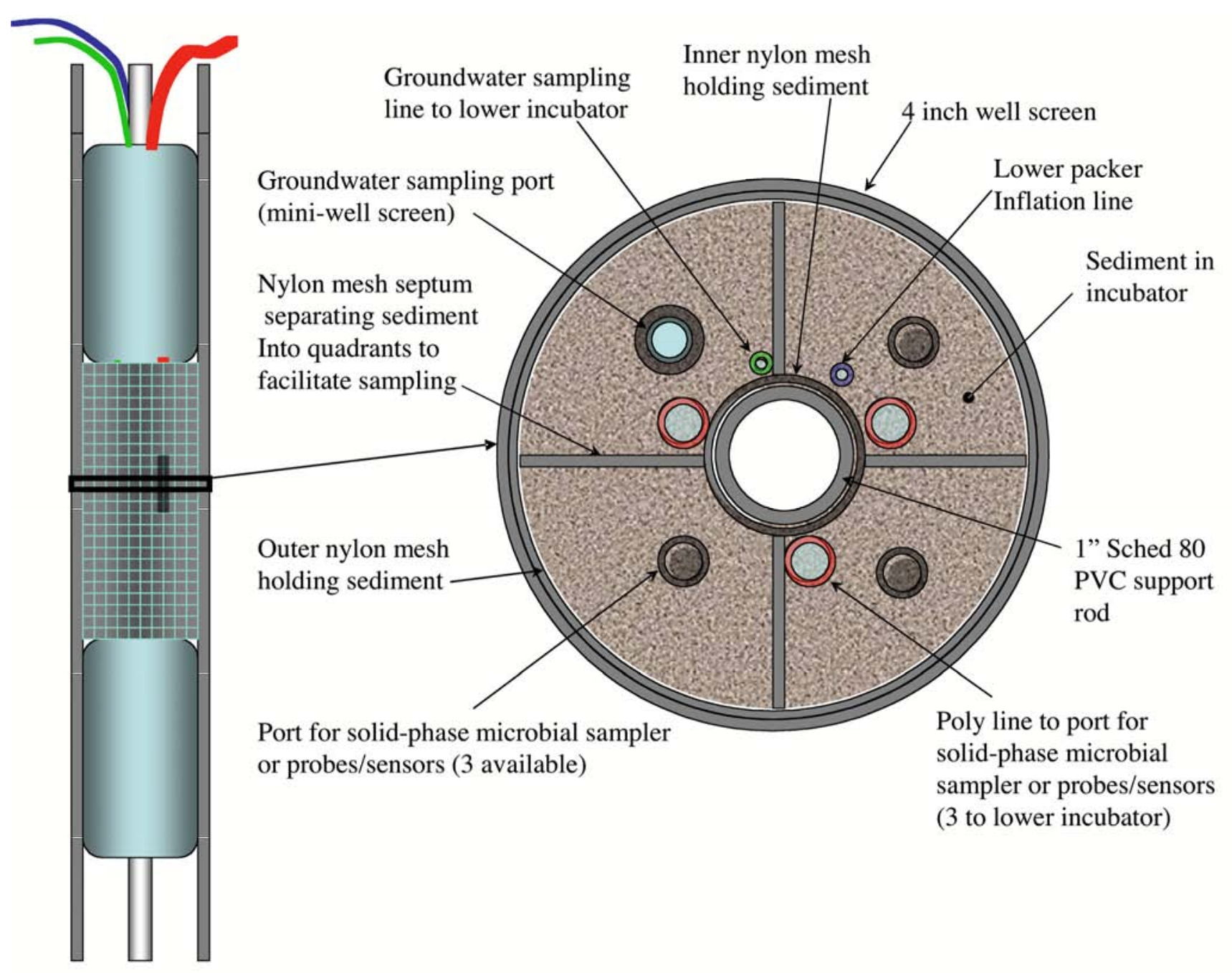

Figure 5. Top view (section) of sediment incubator. 
Sediment for the in situ sediment incubators

Rifle sediment Cobles removed \& mixed 1-3 mm sieved for higher permeability sand-silt

$\mathrm{U}(\mathrm{VI}) 0.17 \mathrm{mg} / \mathrm{L}$ from groundwater during perfusion

Sediment + DIRB + Lactate $\Rightarrow$ SRB DIRB $\$ [Hypothesis 1]

Sediment + Lactate + DIRB vs Sediment + Lactate + SRB measure bioreduced U(IV) [Hypothesis 2]

Sediment $+\mathrm{FeS}_{0.9}$ Sterile (short time) vs SRB + acetate [Hypothesis 3]

Model: Sediment $+\mathrm{FeS}_{09}+$ Lactate SRB + SOB 


\section{Assessing subsurface microbiota}

From Sediment Samplers

DNA 16S rRNA, rDNA,

Genes

DSR (SRB),

DIRB ? NADPH-iron reductase?

soxA sulfite oxidase

by DGGE, Q-PCR, T-RFLP

RNA D. Chandler

Lipids PLFA, Respiratory Quinones, PHA, DMA (Clostridia) ? Spores (DPA) 


\section{Better Respiratory Quinone Assessment \\ Problem present at $\mathrm{mmol} / \mathrm{mol}$ PLFA}

\section{HPLC/electrochemical cell/electrospray/ MS/MS}

HPLC separates components so greater duty cycle

Electrochemical cell $\Rightarrow$ Reduces only Quinones at the specific Eo potential : + $112 \mathrm{mV}$ Ubiquinone; + $36 \mathrm{mV}$ Desmethyl Menaquinone;

- $74 \mathrm{mV}$ Menaquinone

$100 \%$ ionized with - 2 charge $\Rightarrow$ ideal for electrospray ionization

Compare to atmospheric pressure chemical ionization (APCI)

inefficient $\mathrm{H}^{+}$charge transfer from activated gas

MS/MS search for progenitors ions at products: UQ m/z - 197, - 98.5

DMK m/z - 173, -86.5; MK m/z -187 -93.5

Greatly increase sensitivity $\Rightarrow$ greatly increase localization 